\title{
Biomaterial obtenido a partir de micelio de hongo (ganoderma lucidum) y residuos agrícolas
}

\section{Biomaterial obtained from fungus micellium (ganoderma lucidum) and agricultural wastes}

\author{
DOI: $10.46932 / \mathrm{sfjdv2n3-065}$
}

Received in: May 1st, 2021

Accepted in: Jun 30th, 2021

\section{Gutiérrez Díaz Evelyn Susel}

Licenciada en Biología

Tecnológico Nacional de México, Instituto Tecnológico Superior del Oriente del Estado de Hidalgo

Carretera Apan-Tepeapulco, Km 3.5, Col. Las Peñitas, Apan, Hidalgo, México, C.P. 43990

E-mail: 18030761m@itesa.edu.mx

\section{Edna María Hernández Domínguez}

Doctora en Ciencias en Biotecnología

Universidad Politécnica de Pachuca, Hidalgo

E-mail: ednahernandez@upp.edu.mx

\section{Ana Guadalupe Estrada Fernández}

Doctora en Biotecnología

Tecnológico Nacional de México, Instituto Tecnológico Superior del Oriente del Estado de Hidalgo

Carretera Apan-Tepeapulco, Km 3.5, Col. Las Peñitas, Apan, Hidalgo, México, C.P. 43990

E-mail: aestrada@itesa.edu.mx

\section{Jorge Álvarez Cervantes}

Doctor en Ciencias en Biotecnología

Universidad Politécnica de Pachuca, Hidalgo

E-mail: jorge_ac85@upp.edu.mx

\section{Leiry Desireth Romo Medellín}

Maestra en Administración de la Calidad

Tecnológico Nacional de México, Instituto Tecnológico Superior del Oriente del Estado de Hidalgo

Carretera Apan-Tepeapulco, Km 3.5, Col. Las Peñitas, Apan, Hidalgo, México, C.P. 43990

E-mail: lromoQitesa.edu.mx

\section{Bethsua Mendoza Mendoza}

Maestra en Ciencias en Alimentos

Tecnológico Nacional de México, Instituto Tecnológico Superior del Oriente del Estado de Hidalgo

Carretera Apan-Tepeapulco, Km 3.5, Col. Las Peñitas, Apan, Hidalgo, México, C.P. 43990

E-mail: bmendoza@itesa.edu.mx 


\title{
RESUMEN
}

Actualmente la gran cantidad de residuos orgánicos e inorgánicos que se generan en el mundo se están convirtiendo en un problema ambiental. Aunque existen diversas investigaciones para su confinamiento, tratamiento o aprovechamiento, queda mucho por investigar. Desde hace aproximadamente una década, se han desarrollado biomateriales, los cuales son obtenidos a partir de biopolímeros de fuentes vegetales, residuos agroindustriales, así como de bacterias y hongos. El objetivo de la presente investigación es el diseño de un biomaterial a través de la propagación micelial del hongo Ganoderma lucidum, crecido sobre cáscara de nuez y aserrín de roble, ambos residuos agroindustriales. Se formularon cinco sustratos distintos, variando las proporciones de cáscara de nuez (CN) y aserrín (AS) los cuales se describen a continuación; TI-CN $\mathrm{CN}_{100}: \mathrm{AS}_{0}$, TII-CN $\mathrm{CN}_{25}: \mathrm{AS}_{75}$, TIII-CN $\mathrm{CN}_{50}: \mathrm{AS}_{50}, \mathrm{TIV}-\mathrm{CN}_{75}: \mathrm{AS}_{25}$ y TV-CN 0 :AS $\mathrm{A}_{100}$. Después de 30 días de incubación $\left(26^{\circ} \mathrm{C}\right.$, y una humedad relativa del 70 al 80\%), el TI-CN $\mathrm{CN}_{100}: \mathrm{AS}_{0}$, permitió un desarrollo micelial del hongo Ganoderma lucidum. El biomaterial obtenido presentó un aspecto aterciopelado blanquecino con una estructura similar a una espuma. La prueba de compresión reveló que el biomaterial tiene alta resistencia a $0.392 \mathrm{MPa}$, con un porcentaje de deformación máximo de $26 \%$. Además, presentó una densidad media de $0.511 \pm 0.169 \mathrm{~g} / \mathrm{cm}^{-3}$. En general, el biomaterial de estudio se encuentra dentro de los parámetros establecidos para las espumas a base de micelio elaboradas recientemente. Por lo tanto, es un candidato para reemplazar el poliestireno expandido, sin embargo, se requiere una caracterización más amplia, así como estandarización del proceso.

Palabras clave: Biomaterial, Ganoderma lucidum, Residuos agrícolas, Biorremediación.

\begin{abstract}
Currently, the large amount of organic and inorganic wastes generated in the world is becoming an environmental problem. Although there are several researches for their confinement, treatment or utilization, there is still a lot of research to be done. For about a decade, biomaterials have been developed, which are obtained from biopolymers from plant sources, agroindustrial waste, as well as from bacteria and fungi. The objective of the present research is the design of a biomaterial through mycelial propagation of the fungus Ganoderma lucidum, grown on walnut shells and oak sawdust, both agroindustrial wastes. Five different substrates were formulated, varying the proportions of walnut shell $(\mathrm{CN})$ and sawdust (AS) which are described below; TI-CN100:AS0, TII-CN25:AS75, TIII-CN50:AS50, TIVCN75:AS25 and TV-CN0:AS100. After 30 days of incubation $\left(26^{\circ} \mathrm{C}\right.$, and a relative humidity of 70 to 80\%), the TI-CN100:AS0 allowed mycelial development of the fungus Ganoderma lucidum. The biomaterial obtained presented a whitish velvety appearance with a foam-like structure. The compression test revealed that the biomaterial has high strength at $0.392 \mathrm{MPa}$, with a maximum deformation percentage of $26 \%$. In addition, it presented an average density of $0.511 \pm 0.169 \mathrm{~g} / \mathrm{cm}-3$. Overall, the study biomaterial is within the established parameters for recently developed mycelium-based foams. Therefore, it is a candidate to replace expanded polystyrene, however, further characterization and standardization of the process is required.
\end{abstract}

Keywords: Biomaterial, Ganoderma lucidum, Agricultural waste, Bioremediation.

\section{INTRODUCCIÓN}

Alrededor de 50 años, la ciencia de los biomateriales ha sido considerada un campo amplio que define a los biomateriales como materiales biocompatibles (Jemison y Olabisi, 2021). Prodanović y Milutinović (2017), definen biomaterial, como materiales producidos completamente o en parte, por 
materias primas agrícolas (almidón, azúcar, aceite vegetal, celulosa, fibras naturales, paja, entre otros), madera y otras biomoléculas especiales, como la lignina y el caucho natural. Estos materiales son tan versátiles que pueden ser utilizados en el área de la medicina, farmacia, la industria alimentaria, automotriz, textil e incluso la construcción, están destinados a funcionar como soporte o reemplazo de alguna parte en máquinas u órganos del cuerpo humano, por otro lado, pueden servir para la formación de empaques (plásticos biodegradables), envases o materiales de embalaje de alimentos u otros productos. De la misma forma su diseño requiere de enfoques interdisciplinarios que incluyen la ciencia de los materiales, biología, química, médica y mecánica (Basu y Nath, 2009).

La importancia del diseño y caracterización de los biomateriales, radica en su menor impacto al medio ambiente, debido a que pueden ser degradados fácilmente en condiciones ambientales normales, contribuyendo de esta forma en la disminución de la contaminación y afectación de los recursos naturales no renovables. En los últimos años se han desarrollado bioplásticos en forma de recubrimientos, películas, y materiales preformados, que pueden sustituir a los polímeros sintéticos. La materia prima principal para su obtención, son biopolímeros, los cuales pueden ser obtenidos a partir de fuentes vegetales, bacterianas, o fúngicas. Este último, ha sido uno de los campos de investigación con gran potencial biotecnológico, ya que utilizan el micelio de hongos para el desarrollo de biomateriales. El micelio de los hongos está constituido por una red de estructuras celulares filamentosas llamadas hifas ricas en quitina que tiene la capacidad de fusión cuando crecen sobre un determinado sustrato, confiriendo gran resistencia (Attias et al., 2020). Por esta razón, el objetivo de esta investigación fue diseñar un biomaterial a partir del micelio del hongo Ganoderma lucidum crecido sobre cáscara de nuez y aserrín como sustrato. Caracterizarlo mediante pruebas físicas, mecánicas y estructurales para determinar su factibilidad en comparación con las espumas a base de micelio, reportadas en la literatura.

\section{MATERIALES Y MÉTODOS}

El hongo Ganoderma lucidum se adquirió en esporas inoculadas en grano de sorgo (Nanácatl Hongos). La semilla de sorgo inoculado se almacenó a una temperatura de $4^{\circ} \mathrm{C}$ hasta su uso. La cáscara de nuez se adquirió con vendedores de la región de Atotonilco el Grande en el estado de Hidalgo, México y el Aserrín de roble en la región de Tulancingo Hidalgo, México.

\subsection{CARACTERIZACIÓN DEL SUSTRATO}

Se realizó un análisis bromatológico de la cáscara de nuez Carya illinoinensis y del aserrín de Quercus robur. El contenido de humedad (NMX-F-083-1986) y cenizas (NMX-F-066-S-1978) fueron determinadas por diferencia de peso, al igual que fibra cruda después de una digestión ácida y alcalina 
(NMX-F-090-S-1978), contenido de grasas con el método de Soxhlet (NMX-F-089-1978), proteínas por medio del método Kjedahl (NMX-F-068-S-1980) e hidratos de carbono por diferencia.

\subsection{DISEÑO DE MEZCLAS}

Con el programa Minitab® 17.1.0 (Versión 10.0.19041 compilación 19041) se diseñó el experimento para la formulación de mezclas, utilizando distintas proporciones de cáscara de nuez y aserrín. En total se formularon cinco tratamientos (Tabla 1). Cada sustrato fue hidratado 24 horas antes de su utilización (2.5 L de agua/kg de nuez y $3.5 \mathrm{~L} / \mathrm{kg}$ de aserrín) y enriquecido con 90 g de salvado de trigo y $10 \mathrm{~g}$ de sacarosa.

\begin{tabular}{|c|c|c|}
\hline Tratamiento & $\mathrm{CN}(\%)$ & $\operatorname{AS}(\%)$ \\
\hline TI-CN ${ }_{100}: \mathrm{AS}_{0}$ & 100 & 0 \\
\hline TII-CN $25: \mathrm{AS}_{75}$ & 25 & 75 \\
\hline TIII-CN $50: \mathrm{AS}_{50}$ & 50 & 50 \\
\hline TIV-CN ${ }_{75}: \mathrm{AS}_{25}$ & 75 & 25 \\
\hline TV-CN ${ }_{0}: \mathrm{AS}_{100}$ & 0 & 100 \\
\hline
\end{tabular}

Donde: $\mathrm{CN}$ : cáscara de nuez; $\overline{\mathrm{AS}}$ : aserrín. En los tratamientos $\mathrm{T} \#-\mathrm{CN}_{\mathrm{X}}: \mathrm{AS}_{\mathrm{Y}}$ el subíndice X representa la concentración de cáscara de nuez y el subíndice Y la concentración de aserrín.

\subsection{PREPARACIÓN DEL SUSTRATO}

Se llevó a cabo de acuerdo a la metodología propuesta por Sánchez y Royce (2001) con ligeras modificaciones. La cáscara de nuez y el aserrín, fueron triturados con un procesador de alimentos de mano (Oster M2609), se utilizó un juego de tamices (Mon_Motx) de acero inoxidable malla 100 (abertura= $0.149 \mathrm{~mm}$ ). Se prosiguió con la hidratación, enriquecimiento y homogeneización, para ser colocada la mezcla anterior de los tratamientos en bolsas de polipropileno. Se esterilizaron en una autoclave horizontal semiautomática (FELISA modelo FE-399), a una temperatura de $121{ }^{\circ} \mathrm{C}$ durante un tiempo de 15 minutos.

\subsection{FORMACIÓN DEL BIOMATERIAL}

Como primera etapa, se llevó a cabo la inoculación del de G. lucidum en condiciones asépticas. 10 $\mathrm{g}$ de semilla de sorgo con esporas del hongo fueron mezcladas con $62 \mathrm{~g}$ de sustrato, fueron colocadas en cajas petri de plástico redondas de 100 x 15 mm. Esta operación se realizó para los cinco tratamientos (TI$\mathrm{CN}_{100}: \mathrm{AS}_{0}$, TII-CN $\mathrm{CN}_{25}: \mathrm{AS}_{75}$, TIII-CN $\mathrm{CN}_{50}: \mathrm{AS}_{50}, \mathrm{TIV}-\mathrm{CN}_{75}: \mathrm{AS}_{25}$ y TV-CN 0 :AS 100 ). Posteriormente, en una estufa de cultivo digital (FELISA modelo FE-399) se incubaron por un tiempo de 30 días a una temperatura de $26^{\circ} \mathrm{C}$, manteniendo una humedad relativa de 70 a $80 \%$. Finalmente, para los tratamientos 
donde se observó que el micelio creció de forma homogénea y logró compactar el sustrato, se sometió a un tratamiento de secado, retirando de la caja petri y dejando al sol por 72 horas.

\subsection{CARACTERIZACIÓN DEL BIOMATERIAL}

Como parte de la caracterización física del biomaterial se realizaron las pruebas de Microscopia Electrónica de Barrido (SEM) y morfología micelial por medio de la toma de micrografías. Para el cálculo del tamaño de las hifas y de los basidiomicetos se llevó a cabo una preparación previa la cual consistió en el cubriendo una pequeña porción de la muestra de la matriz del biomaterial con oro en un equipo BALTEC SCD 050 Sputter Coater. Posteriormente se realizó la toma de micrografías en un equipo JEOL JSM7600 F Field Emission Scanning Electron Microscope.

Las principales características de la morfología micelial como textura (algodonosa o flocosa), densidad (alta, regular o baja), color (blanquecino, blanco o rosa pálido) y crecimiento (escaso, regular o abundante) se identificaron mediante observación visual después de la colonización completa de las placas de Petri, de acuerdo con lo establecido por Sobal et al., (2007).

Las Propiedades mecánicas se midieron con una prueba de compresión de 1 ciclo utilizando una prensa universal (Shimadzu UH-500 KNI), a una velocidad de $1 \mathrm{~mm} / \mathrm{s}$, determinando el esfuerzo máximo en $\mathrm{kgf} / \mathrm{mm}^{2}$ soportado por el material antes de la ruptura. El material fue cortado en cilindros de $50 \mathrm{~mm}$ de diámetro por $8.5 \mathrm{~mm}$ de altura.

La densidad del material fue determinada, tomando tres porciones de 3 x $3 \mathrm{~cm}$, fueron pesados y colocados en agua con una probeta graduada, para registrar el volumen de agua desplazado al introducir el material. Finalmente, los cálculos fueron llevados de acuerdo a la ecuación 1.

$$
\text { Densidad }=\frac{\text { masa }}{\text { volumen }} \quad \text { Ec. } 1
$$

\subsection{ANÁLISIS ESTADÍSTICO}

Todos los análisis fueron llevados a cabo por triplicado, se calculó la media y la desviación estándar para cada medición. Los datos fueron analizados con una prueba $t$-student para comparación de dos medias o contra un valor de referencia utilizando una $p \leq 0.05$. Se utilizó en programa Sigma Plot (Versión 12.0 Build 12.2.0.45). 


\section{RESULTADOS Y DISCUSIÓN}

\subsection{CARACTERIZACIÓN DEL SUSTRATO}

Los resultados del análisis bromatológico a la cáscara de nuez se presentan en la Tabla 2, se observa que el componente principal es la fibra cruda con un $45.2 \%$, seguido de los carbohidratos (44.5\%), humedad $(4.51 \%)$, cenizas $(2.63 \%)$, proteína $(2.5 \%)$ y grasa $(0.77 \%)$. Do Prado et al. (2013), destaca que la composición química de la cáscara de nuez varía con la temporada del año, sin embargo, reportan valores de $48.87 \%$ de fibra cruda y $11.3 \%$ de humedad, estos valores son mayores a los obtenidos en el presente trabajo. En contraparte, los valores para carbohidratos son menores $34.57 \%$ a los encontrados en la nuez de estudio. Finalmente, reporta trazas de proteínas y grasas concordando con los resultados obtenidos. García y Serrano, (2015) mencionan que en México la cáscara de nuez es almacenada por largos periodos de tiempo y por lo tanto se considera conveniente que tenga valores entre 4 y $4.5 \%$ de humedad, lo cual, facilita su almacenamiento y alarga su vida útil. De acuerdo, con los resultados bromatológicos obtenidos para la cáscara de nuez, esta presenta los nutrientes necesarios para realizar las mezclas y proporcionar un medio de cultivo adecuado para el crecimiento del hongo.

Tabla 2. Resultados del análisis bromatológico a la cáscara de nuez

\begin{tabular}{ccc}
\hline Análisis & Valor promedio $(\%)$ & $\begin{array}{c}\text { Referencia (\%) } \\
\text { Do Prado et al., 2013 }\end{array}$ \\
\hline Humedad & $4.51 \pm 0.49^{\mathrm{a}}$ & $11.30^{\mathrm{b}}$ \\
Ceniza & $2.63 \pm 0.12^{\mathrm{a}}$ & $1.85^{\mathrm{b}}$ \\
Proteínas & $2.50 \pm 0.42^{\mathrm{a}}$ & $2.84^{\mathrm{a}}$ \\
Carbohidratos & $44.50 \pm 0.48^{\mathrm{a}}$ & $34.57^{\mathrm{b}}$ \\
Grasa & $0.77 \pm 0.40^{\mathrm{a}}$ & $0.39^{\mathrm{a}}$ \\
Fibra cruda & $45.00 \pm 0.44^{\mathrm{a}}$ & $48.47^{\mathrm{b}}$
\end{tabular}

Valores promedio de cinco repeticiones \pm la desviación estándar. ${ }^{\text {abc }}$ letras diferentes en la misma fila, existen diferencias estadísticamente significativas $\mathrm{P}(<0.05)$.

Para el aserrín se determinaron humedad y cenizas. Los valores obtenidos de fueron de $14.11 \% \pm$ 0.93 y $0.20 \% \pm 0.13$, respectivamente. En el estudio realizado por Forero, Camargo y Sierra (2014), reportan $10.01 \%$ de humedad y $2.88 \%$ de cenizas; Serret, Giralt y Quintero (2016), mencionan valores de 7.83 a $9.73 \%$ de humedad y para cenizas de 0.79 a $0.89 \%$, valores que resultan inferiores a los reportados en el presente trabajo y a los que indica Levelton, (1975), 25 a $40 \%$ de humedad y de 0.5 a $2.0 \%$ de cenizas (Tabla 3). Los porcentajes reportados por CONAFOR, (2010) fueron de 10 y $0.2 \%$ respectivamente para humedad y cenizas, además, menciona que contiene de $65 \%$ de carbohidratos, del 3 al $7 \%$ de grasa y $20 \%$ 
de fibra cruda. Por lo tanto, el aserrín utilizado en esta investigación, tiene los nutrientes necesarios para realizar las mezclas de los tratamientos.

\subsection{FORMACIÓN DEL BIOMATERIAL}

El crecimiento micelial de G. lucidum fue favorable después de un tiempo de 30 días de incubación en el tratamiento TI-CN $\mathrm{CN}_{100}: \mathrm{AS}_{0}$ con $100 \%$ de cáscara de nuez (C. illinoinensis), enriquecido con salvado de trigo y sacarosa. Para el cultivo de G. lucidum se utilizan sustratos de maderas duras, generalmente, del género Quercus sp., (Rodríguez y Jaramillo, 2005). Sin embargo, no se logró formar el material en los tratamientos TII-CN $\mathrm{CN}_{25}: \mathrm{AS}_{75}$, TIII-CN $\mathrm{CN}_{50}: \mathrm{AS}_{50}, \mathrm{TIV}-\mathrm{CN}_{75}: \mathrm{AS}_{25}$ y TV-CN 0 : $\mathrm{AS}_{100}$ los cuales contenían un porcentaje de aserrín de Quercus sp ya que el micelio no logró unirse a el sustrato, solo se desarrolló micelio aéreo, dejando suelta la parte media del sustrato. Al final del proceso el material previamente seco tuvo un peso de $30 \mathrm{~g}$, cuya reducción de peso fue de 58.4\% (Figura 1). La generación de biomateriales inició al inocular el micelio de hongos de podredumbre blanca en sustratos orgánicos sólidos de naturaleza lignocelulósica. Al transcurrir el tiempo, y bajo condiciones controladas de crecimiento, el material orgánico se degradó y reemplazó por la biomasa fúngica, creando una capa esponjosa o compacta la cual entreteje al sustrato, se moldea o adhiere a soportes naturales y sintéticos. Al final, se obtuvo un compuesto ligero, que consistió en una red entrelazada tridimensionalmente de fibras naturales presentes en la materia prima y células miceliales filamentosas que, como adhesivo natural, otorgan una elevada resistencia mecánica y flexibilidad estructural al biomaterial obtenido (Feijóo et al., 2021).

Tabla 3. Resultado de análisis bromatológico del aserrín (Quercus robur)

\begin{tabular}{lcccc}
\hline \multicolumn{1}{c}{ Análisis } & $\begin{array}{c}\text { Valor promedio } \\
(\%)\end{array}$ & $\begin{array}{c}\text { Forero, Camargo y } \\
\text { Sierra, 2014. }\end{array}$ & $\begin{array}{c}\text { Serret, Giralt y } \\
\text { Quintero, 2016. }\end{array}$ & $\begin{array}{c}\text { CONAFOR, } \\
\mathbf{2 0 1 0}\end{array}$ \\
\hline Humedad & $14.11 \pm 0.93^{\mathrm{a}}$ & $10.01^{\mathrm{b}}$ & $7.83-9.73^{\mathrm{b}}$ & $10^{\mathrm{b}}$ \\
Ceniza & $0.20 \pm 0.13^{\mathrm{a}}$ & $2.88^{\mathrm{b}}$ & $0.79-2.0^{\mathrm{b}}$ & $0.2^{\mathrm{a}}$ \\
Carbohidratos & --- & & 65 \\
Grasa & --- & & $3-7$ \\
Fibra cruda & --- & & 20 \\
\hline
\end{tabular}

Valores promedio de cinco repeticiones \pm la desviación estándar. ${ }^{\text {abc }}$ letras diferentes en la misma fila, existen diferencias estadísticamente significativas $\mathrm{P}(<0.05)$. 
Figura 1. a) vista lateral de biomaterial formado con el tratamiento TI- $\mathrm{CN}_{100}: \mathrm{AS}_{0}$; b) vista frontal del biomaterial formado

a)

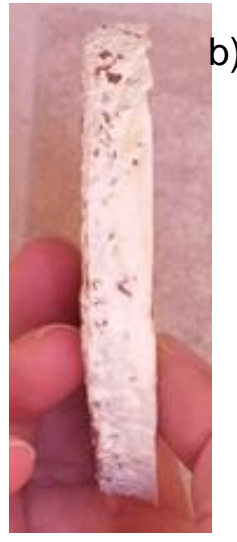

b)

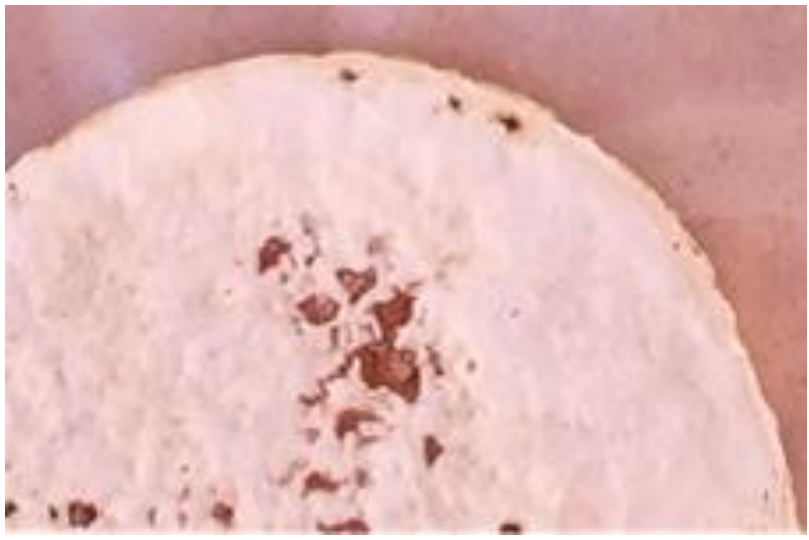

Diversos análisis de experimentación asumen que son pocos los parámetros, pero, esenciales para el crecimiento del hongo y el desarrollo adecuado del micelio; hacen referencia a la capacidad de absorción de agua de los sustratos alrededor del 65 a 75\%, nivel del pH inicial de 6 (Bidegain, Cubitto y Curvetto, 2015), contenido de nitrógeno del sustrato inicial en niveles más altos o cercanos al 1\%. El pH de cada uno de los sustratos al inicio de la inoculación osciló entre 5.50 a 6.24 y específicamente para el tratamiento TI-CN $\mathrm{CN}_{100}: \mathrm{AS}_{0}$ el pH fue de 5.5, sin embargo, la invasión micelial completa de G. lucidum se presentó sin ningún inconveniente. Este resultado coincide con los reportado por González-Matute et al., (2002), reportan que la tasa de crecimiento mayor se presentó a pH de 4.90 y 5.60. Los tratamientos con aserrín TIV-CN75: $\mathrm{AS}_{25}$ y TV-CN $0: \mathrm{AS}_{100}$ presentaban el $\mathrm{pH}$ óptimo, y no hubo invasión completa de micelio, Attias et al., (2017) considera, que no hay una relación coherente entre el pH y el desarrollo de micelio.

En cuanto a la relación Carbono/Nitrógeno $(\mathrm{C} / \mathrm{N})$ de los sustratos se sabe que tanto la cáscara de nuez C. illinoinensis y el aserrín de $Q$. robur cuentan con los carbohidratos necesarios, ya que se tomó el $75 \%$ de cáscara de nuez y de aserrín, los cuales aportan una fuente de carbono, el $22.5 \%$ de salvado de trigo que aporta la fuente de nitrógeno y el $2.5 \%$ de sacarosa, tomando en cuenta la propuesta de Miles y Chang (1997). Torres et al., (2011) menciona que para G. lucidum la relación C/N no afecta significativamente la producción de biomasa a concentraciones altas, mientras que a concentraciones bajas la relación $\mathrm{C} / \mathrm{N}$ sí la afecta. Manteniendo constante la relación $\mathrm{C} / \mathrm{N}$, la concentración de biomasa de $G$. lucidum se incrementa con el aumento de la fuente de carbono. Por otro lado, para mejorar la calidad nutricional de un sustrato se está utilizando la aplicación de otros aditivos como aceites vegetales los cuales incentivan el crecimiento de los basidiomas (Suárez y Nieto, 2013; Yang, Ke y Kuo, 2000). 


\subsection{CARACTERIZACIÓN DEL BIOMATERIAL}

En la figura 2, pueden observarse las micrografías del tratamiento $\mathrm{TI}_{-} \mathrm{CN}_{100}: \mathrm{AS}_{0}$. Se pueden percibir hifas generativas con un diámetro de unión de 0.75 a $3 \mu \mathrm{m}$ (Figura 2a) y presencia de conidios. En la figura $2 \mathrm{~b}$, se observa la presencia de basidios con dimensiones de $9.6 \mu \mathrm{m}$ de alto por $8.5 \mu \mathrm{m}$ de ancho y de $13.7 \mu \mathrm{m}$ de alto por $6.6 \mu \mathrm{m}$ de ancho. Y en la figura $2 \mathrm{c}$ se observa un sistema hifal dimítico con estructuras productoras de basidioesporas, sobre el tratamiento de cáscara de nuez.

Las micrografías del tratamiento $\mathrm{TV}-\mathrm{CN}_{0}: \mathrm{AS}_{100}$, se observan hifas generativas con un diámetro de 1 - $1.25 \mu \mathrm{m}$, sobre el sustrato de aserrín (Figura 3a). Sistema hifal dimítico; hifas generativas, con uniones de 0.76 - $1.92 \mu \mathrm{m}$ de diámetro; hifas esqueléticas (Figura 3b) abundantes, de 3 - $6 \mu \mathrm{m}$ de diámetro. Se observan basidios de $13 \mu \mathrm{m}$ de alto por $6.8 \mu \mathrm{m}$ de ancho y de $13 \mu \mathrm{m}$ de alto por $6 \mu \mathrm{m}$ de ancho en un sistema hifal dimíco invadiendo el sustrato de aserrín (Figura 3c). En la última micrografía (Figura 3d) se observa el micelio de G. lucidum invadiendo el sustrato de aserrín.

Figura 2. Micrografías de Electrónica de barrido del biomaterial con tratamiento de C. illinoinensis. En la imagen 16 a) 3,000x hifas generativas con un diámetro de unión de 0.75 a $3 \mu \mathrm{m}$. b) 2,000x basidios el de la parte superior con una dimensión de 9.6 x $8.5 \mu \mathrm{m}$ y el de la parte inferior con dimensiones de 13.7 x $6.6 \mu \mathrm{m}$. c) 1,000x invasión del micelio con estructuras productoras de basidioesporas, sobre el tratamiento de cáscara de nuez.
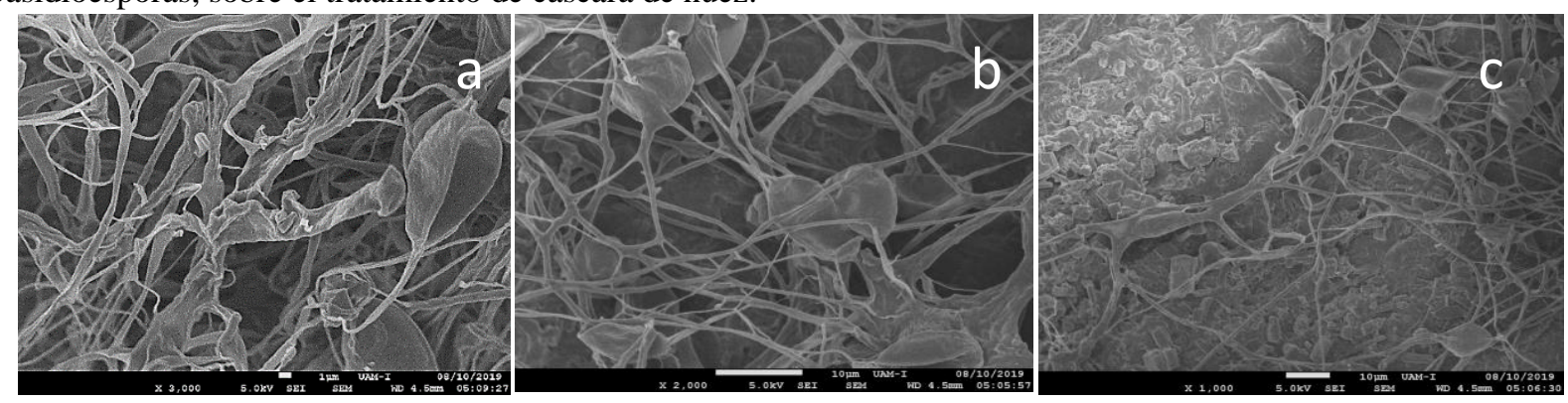
Figura 3. Micrografías en Microscopio Electrónico de Barrido de biomaterial con tratamiento de aserrín Quercus robur. En la imagen 18a) 5,500x hifas generativas con un diámetro de 1 a $1.25 \mu \mathrm{m}$, sobre el sustrato de aserrín b) 1,700x sistema hifal dimítico con hifas generativas e hifas esqueléticas con uniones de 0.76 a $1.92 \mu \mathrm{m}$ de diámetro c) $1,100 \mathrm{x}$ basidios de $13 \mu \mathrm{m}$ de alto por $6.8 \mu \mathrm{m}$ de ancho y d) 900x invasión de micelio de G. lucidum sobre el sustrato de aserrín.
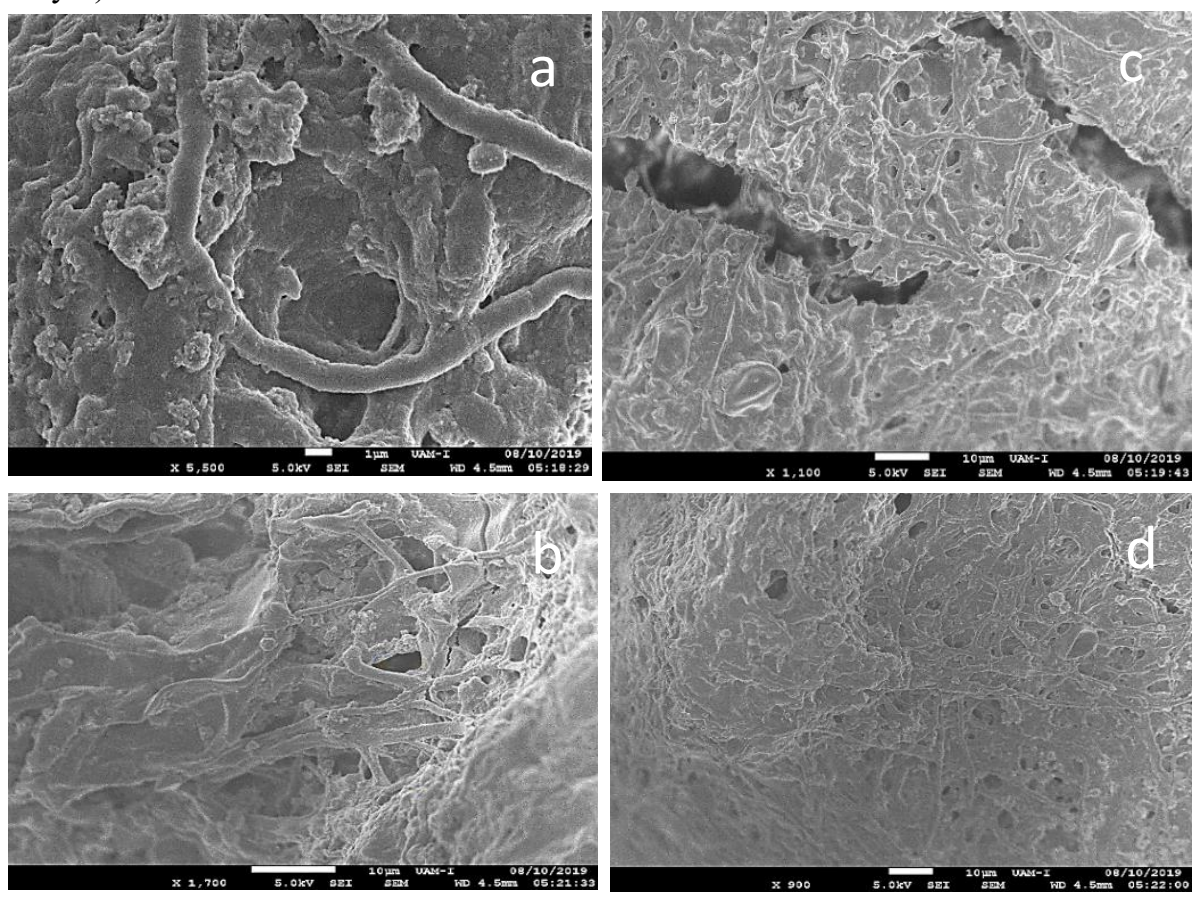

El tamaño promedio de las hifas del tratamiento TI-CN $\mathrm{CN}_{100}$ : $\mathrm{AS}_{0}$ fue de $2.25 \pm 1.74 \mu \mathrm{m}$ y para el tratamiento $\mathrm{TV}-\mathrm{CN}_{0}: \mathrm{AS}_{100}$, el tamaño promedio de las hifas fue de $1.29 \pm 0.40 \mu \mathrm{m}$. El micelio es el crecimiento vegetativo de hongos filamentosos, formado por una red de microfilamentos con diámetros que oscilan entre 1 - $30 \mu \mathrm{m}$, según el tipo de especie y el entorno de crecimiento (Islam et al., 2017). La fisiología del micelio es un determinante clave de la fuerza y las características del producto final. Las paredes celulares de los micelios están compuestas de quitina, el mismo biopolímero que los cuerpos de los insectos y las langostas (Zellery y Zocher, 2012). Para ambos tratamientos se midió el crecimiento micelial después de un periodo de tiempo de 14 días de colonización, se observó en las micrografías las paredes celulares de los sustratos cubiertas por una red fibrosa de hifas fúngicas. El micelio creció en sustratos orgánicos a través de la expansión de la punta apical de las hifas de una espora o inóculo, en condiciones ambientales (Fricker, Boddy y Bebber, 2007). Las colonias de hifas interactuaron aleatoriamente a través de la fusión de hifas (anastomosis) para formar una estructura de red de fibras (Glass, Rasmussen, Roca y Read, 2004), que actuó como un adhesivo de autoensamblaje natural para unir los sustratos y formar un material compuesto. De acuerdo con los resultados obtenidos en cuanto al diámetro de las hifas en los tratamientos TI-CN $\mathrm{CN}_{100}: \mathrm{AS}_{0}$ y TV-CN $\mathrm{AS}_{100}$ después de un tiempo de 14 días de incubación, existió una variación en cuanto al tamaño de las hifas entre cada uno de los tratamientos. Matos et al., (2019) y Kuijk et al., (2017) coinciden en que las variaciones en el crecimiento micelial de los tratamientos evaluados por diámetro pueden ser debido al tipo de residuo agrícola utilizado como 
sustrato de cultivo para la realización del material. Por otro lado, Islam et al., (2018) considera que el tamaño de partículas de residuos agrícolas utilizadas como sustrato para la producción del biocompuesto está muy relacionado con la disponibilidad de las macromoléculas para el crecimiento micelial. En los resultados obtenidos en la experimentación de Arifin y Yusuf (2013), aclaran que la fuente de carbono proporcionó tanto la estructura como la energía a la célula del micelio, la fuente de carbono fue la más comúnmente prevista para el crecimiento del micelio. Esto indicó que el tipo de sustrato, y el tamaño de partícula, son factores importantes para el crecimiento micelial de cada uno de los materiales elaborados. En la investigación realizada por Attias et al., (2017), mencionan algunos parámetros que son esenciales para alcanzar el crecimiento y desarrollo del micelio, de acuerdo con esto los materiales de base biológica existe una clara correlación entre el cambio cuantitativo en el contenido de la materia orgánica durante el crecimiento de los hongos con los parámetros cualitativos de densidad y espesor de micelio. Es importante asegurarse de la digestión de la materia orgánica para el desarrollo del micelio.

Con respecto a los cambios físicos durante la incubación se observó que en la etapa inicial de la descomposición del aserrín hubo un cambio de coloración en los sustratos que contenían aserrín, la coloración del sustrato se volvió más oscuro las hifas del hongo penetraron en la madera, se inició la colonización y se liberaron enzimas. Lekounougou et al., (2008), señalan que este cambio de color marrón es atribuido a la síntesis de melanina en la etapa temprana de la descomposición de la madera, y se correlaciona con la degradación de los extractos de madera que requieren actividad de lacasas, mientras que los otros sistemas de degradación de la madera (peroxidasas y polisacáridos hidrolasas) aún están reprimidos. Por otro lado, Powrie y Wu (1986), atribuyen que el pardeamiento del material puede ser causado por reacciones de Maillard que involucran azúcares y proteínas presentes en las paredes celulares de los hongos y el material vegetal o alternativamente, puede ser causado por la caramelización de azúcares vegetales y fúngicos o por pirólisis de material orgánico.

En cuanto a la textura micelial, los cinco tratamientos presentaron textura algodonosa, excepto en el tratamiento TI-CN $\mathrm{CN}_{100}: \mathrm{AS}_{0}$ que aunado a esta presentó textura aterciopelada, esto se evaluó a partir del día 10 de incubación. En la densidad de micelio se observó menor en los tratamientos TII-CN $25: \mathrm{AS}_{75}$, TIII-CN $50: \mathrm{AS}_{50}, \mathrm{TIV}-\mathrm{CN}_{75}: \mathrm{AS}_{25}$ y TV-CN $\mathrm{CN}_{100}$ con textura algodonosa regular, en comparación con el tratamiento TI-CN $\mathrm{CN}_{100}: \mathrm{AS}_{0}$. El color del micelio que se observó en todos los tratamientos fue blanquecino. El crecimiento del micelio se inició a partir de los 5 días y fue abundante en el tratamiento TI-CN ${ }_{100}: \mathrm{AS}_{0}$ a lo largo de los 30 días de incubación, en el resto de los tratamientos el crecimiento fue regular a los 10 días, sin embargo, al término de los días de incubación se observó que no hubo invasión completa en el resto de los tratamientos (TII-CN $\mathrm{CN}_{25}: \mathrm{AS}_{75}$, TIII-CN $\mathrm{CN}_{50}: \mathrm{AS}_{50}, \mathrm{TIV}-\mathrm{CN}_{75}: \mathrm{AS}_{25}$ y TV-CN $0: \mathrm{AS}_{100}$ ). La morfología de las colonias de las cepas de G. lucidum fue muy similar con respecto a la morfología de las 
colonias que describe la investigación de Sobal et al., (2007) excepto por la textura del tratamiento TI$\mathrm{CN}_{100}: \mathrm{AS}_{0}$ (algodonosa/aterciopelada) la cual, presentó doble textura.

Existen algunas variables en el desarrollo de la fase experimental para explicar el rendimiento del crecimiento y desarrollo micelial. Uno de ellos es la composición química de los sustratos, ya que si bien, se sabe que el aserrín es uno de los sustratos más aconsejados para el crecimiento y desarrollo de los macro hongos, este, no obtuvo el crecimiento deseado. Los hongos que realizan la descomposición de sustratos requieren de una mayor fuente de carbono que nitrógeno para obtener un ambiente óptimo para su crecimiento y desarrollo. Algunas acciones que se pueden tomar para obtener mejores resultados, es aumentar el área de superficie de los componentes del sustrato reduciendo el tamaño de partícula a alrededor de $2 \mathrm{~mm}$, mantener condiciones aeróbicas en el sustrato y agregar materiales adicionales con mayor capacidad de absorción de agua (Danay et al., 2012). Attias et al., (2017) recomiendan un tratamiento térmico previo para abrir y expandir las cavidades de aire entre las fibras de los sustratos, con el fin de aumentar la porosidad de los sustratos de las formulaciones.

Existe metodología en donde el molde o el recipiente para la elaboración del biomaterial está hecho con madera, Arifin y Yusuf (2013), reportan la utilización de recipientes de polipropileno, lo cual, es más conveniente ya que los moldes de madera también absorben humedad lo cual se cree que interfiere en el desarrollo de micelio y en el secado del material, ya que no es homogéneo. Existen algunas razones posibles para explicar la diferencia del bajo rendimiento entre los tratamientos elaborados, al realizar la inoculación de la semilla de sorgo con micelio de G. lucidum en cada tratamiento se puede deducir que no se distribuyó adecuadamente las partículas de micelio. Algunas áreas de la superficie de las mezclas de los sustratos estaban cubiertas con hifas miceliales, mientras que, otras partes se encontraban menos cubiertas. Es probable, que esta distribución desigual de la semilla de sorgo con micelio sea al menos parcialmente responsable del bajo rendimiento del desarrollo del micelio. Sun et al., (2019) mencionan las siguientes razones: (1) la mejor dispersión del micelio, lo que proporciona una mejor adhesión; (2) los cambios químicos de las partículas de los sustratos, como la degradación de la hemicelulosa y la lignina, que pueden abrir más poros en la superficie de la pared celular de la madera, aumentar la energía de la superficie de la pared celular de los sustratos, aumentar la energía de la superficie y proporcionar más grupos funcionales para la unión y (3) las diferencias químicas en la estructura del micelio cultivado en los diferentes sustratos. Se conoce que en la etapa inicial de descomposición de los sustratos y en especial en madera, las hifas fúngicas crecen en las paredes y la cubierta de las células de madera, las enzimas típicamente producidas por hongos de podredumbre blanca pueden degradar la lignina y producir radicales que podrían ayudar a mejorar la unión adhesiva (Sun et al., 2019). 


\subsection{PROPIEDADES MECÁNICAS}

Los materiales a base de micelio fúngico no cuentan con normas propias (Rodríguez, 2018), por ello, se comparó el esfuerzo de compresión, tensión y densidad de los biomateriales con otros materiales reportados en la literatura. La muestra fue sometida a una carga de $200 \mathrm{kgf}$, en la figura 4 se puede observar que el esfuerzo máximo soportado por el material, está alrededor de $100 \mathrm{kgf}$, o un esfuerzo de 0.04 $\mathrm{kgf} / \mathrm{mm}^{2}(0.392 \mathrm{Mpa})$, ya que la variación en la gráfica indica una ruptura de la estructura del material. La curva de tensión-deformación es bastante lineal, con resistencia alta, lo que indica una fractura progresiva.

Figura 4. Gráfica de esfuerzo contra porcentaje de deformación del biomaterial formado con con el tratamiento TI-CN $100: \mathrm{AS}_{0}$.

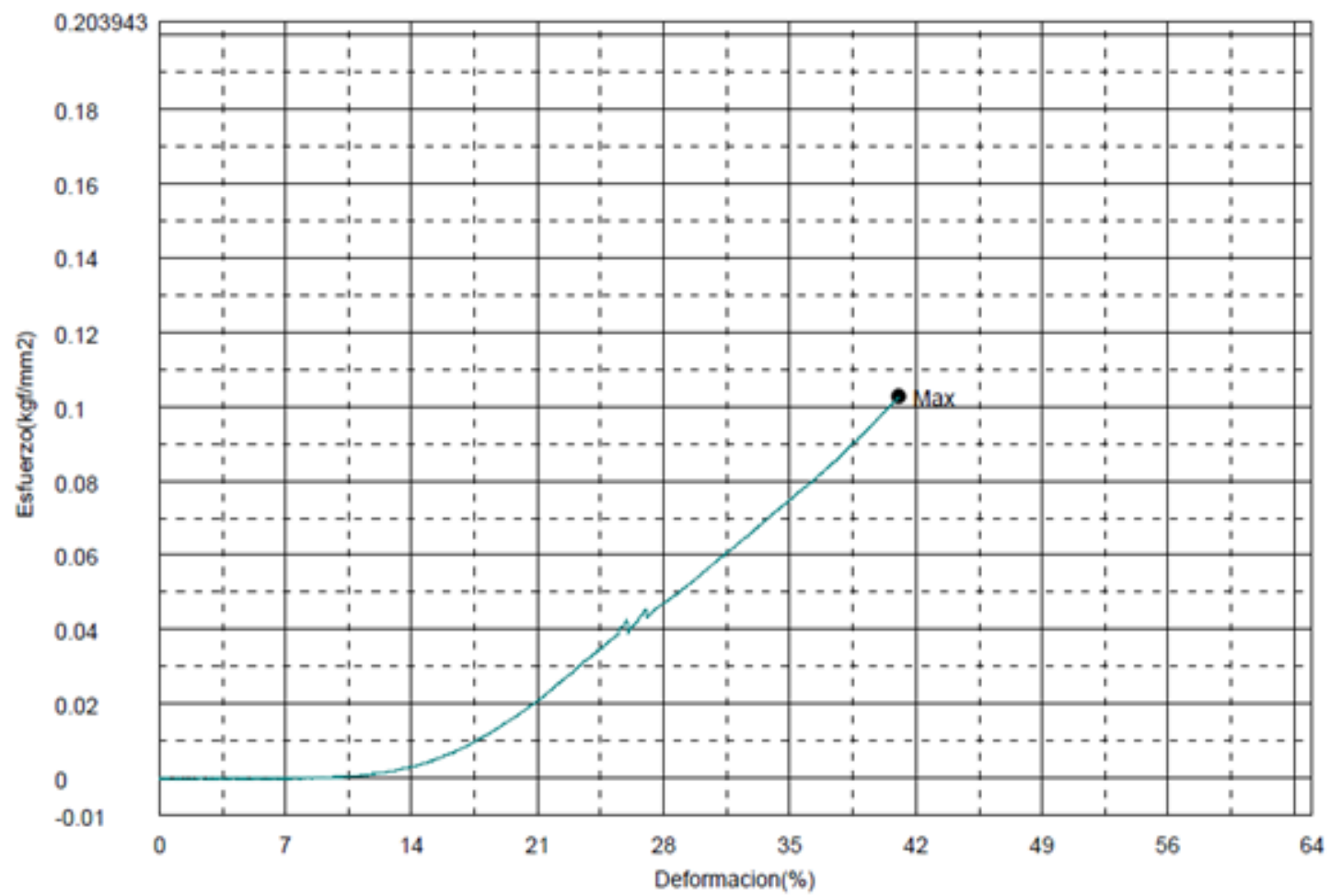

En la investigación realizada por Appels et al., (2019) realizaron biomateriales prensados con calor, prensados en frío y sin prensar con dos tipos de hongos, las muestras prensadas en caliente tuvieron una resistencia a la tracción y un módulo elástico significativamente más altos, en comparación con sus correspondientes de muestras prensadas en frío y no prensadas. El material de paja de colza (PRH) era rígido y fuerte pero quebradizo, como lo mostró su curva pronunciada, alta resistencia a la tracción (0.24 $\mathrm{MPa}$ ). Haciendo una comparación de resistencia a la fractura con el material obtenido en la presente investigación con G. lucidum y cáscara de nuez tuvo un esfuerzo de $0.04 \mathrm{kgf} / \mathrm{mm}^{2}$ (0.392 Mpa), que fue mayor a la elaborada por los autores antes mencionados en sus biomateriales prensados en frío y en calor cultivados en algodón. Según la literatura, la resistencia a la compresión en las espumas a base de micelio 
(MBF) varía constantemente entre $29 \mathrm{kPa}(0.029 \mathrm{MPa})$ y $567 \mathrm{kPa}(0.567 \mathrm{MPa})$, el rango más amplio informado por Amtislavski y Yang (2017). De acuerdo, con la información el biomaterial a base de $G$. lucidum y cáscara de nuez C. illinoinensis está dentro del rango con 0.392 Mpa. Haneef et al., (2017) proporcionó una investigación general sobre las propiedades físicas y mecánicas, sólo que se centró en Pleurotus ostreatus. Según los autores, el sustrato afecta fuertemente la composición micelial en polisacáridos, lípidos y quitina, así como, la morfología general y las propiedades mecánicas. Por otro lado, informan menor alargamiento en la fractura para $P$. ostreatus (4-9 \%) en comparación con $G$. lucidum (14 - 33\%), ya que la mayor fracción proteica y lipídica en G. lucidum actuaría como plastificante. El citado autor se refiere básicamente a proteínas estructurales. Islam et al., (2017) reportó para su material una deformación de aproximadamente el $25 \%$ de acuerdo con su curva de esfuerzo-deformación, desarrolló una torsión aproximadamente en $8 \%$ de deformación. El porcentaje de deformación máximo que se obtuvo en la presente investigación es de $26 \%$, con el desarrollo de una torsión aproximadamente en $11 \%$ de deformación

\subsection{DETERMINACIÓN DE DENSIDAD}

Se sugiere a las espumas base de micelio (MBF) como competencia o sustitutos de las espumas tradicionales de poliestireno y poliuretano. Ya que ofrecen una alternativa sostenible para embalaje. A pesar de que la producción sigue siendo limitada para unas pocas empresas en el mundo, el proceso productivo está mejorando rápidamente, resultando alta calidad de materiales a costos razonablemente bajos La baja densidad se considera un factor importante en la competitividad de las MBF. Junto a la conveniencia de un peso reducido para el envasado, la densidad afecta a otras propiedades importantes del material, tanto desde el punto de vista físico-mecánico como termodinámico (Girometta et al., 2019). El control de la densidad y su homogeneidad dentro del material siguen siendo aspectos problemáticos en MBF, ya que está fuertemente relacionado con la composición y estructura del sustrato. Lógicamente, cuanto mayor es la fracción de granos en el sustrato, mayor es la densidad final en comparación con los protocolos que aumentan la fracción de fibras, cascarillas o pulpa de madera (Arifin y Yusuf, 2013). Dado que, la densidad de las fibras naturales oscila entre 1.2 y $1.5 \mathrm{~g} / \mathrm{cm}^{-3}$ y la densidad de la madera está entre $0.3 \mathrm{y} 0.88 \mathrm{~g} / \mathrm{cm}^{-3}$. El tablero de fibra tiene una densidad media de $0.50 \mathrm{a} 1.00 \mathrm{~g} / \mathrm{cm}^{-3}$, el tablero de fibra orientada (OSB) compuesto de madera tiene una densidad de 0.55 a $0.70 \mathrm{~g} / \mathrm{cm}^{-3}$ (Appels et al., 2019). Es interesante, mencionar que Islam et al., (2017) reportaron una densidad de 0.03 a $0.05 \mathrm{~g} / \mathrm{cm}^{-3}$ para un biomaterial novedoso hecho solo de micelio comprimido. El biomaterial obtenido en esta investigación con cáscara de $C$. illinoinensis tuvo una media de $0.511 \pm 0.169 \mathrm{~g} / \mathrm{cm}^{-3}$, lo cual, indica que su densidad es baja. 


\section{CONCLUSIÓN}

El crecimiento de micelio de Ganoderma lucidum inoculado en el tratamiento TI-CN ${ }_{100}: \mathrm{AS}_{0}$ de cáscara de nuez Carya illinonensis proporcionó las condiciones para el diseño de un biomaterial bajo un método de fabricación biológica de bajo costo para reciclar los residuos de la cáscara de nuez. Se evaluó el aspecto visual, la densidad, las propiedades mecánicas, el biomaterial tuvo un aspecto aterciopelado blanquecino con una estructura similar a una espuma, las propiedades estructurales de los compuestos a base de micelio dependen de la naturaleza del sustrato y del tipo de aditivos utilizados para reforzar el material, además, del tipo de hongo, el ambiente durante la colonización y el procesamiento posterior a la síntesis. En las pruebas mecánicas realizadas, en la prueba de esfuerzo de compresión se determinó que tiene una resistencia alta a la compresión, con un porcentaje de deformación máximo de $26 \%$, presentó una densidad media de $0.511 \pm 0.169 \mathrm{~g} / \mathrm{cm}^{-3}$ lo cual, se considera como baja. En general, el biomaterial obtenido está dentro de los parámetros de las espumas a base de micelio elaborados más recientemente, es rígido, esta característica de resistencia a la compresión está influenciada de manera apreciable por su densidad, presentó una baja densidad lo cual es beneficioso para este tipo de materiales y al ser biodegradable, ofrece una alternativa ecológica a los materiales de embalaje a base de petróleo. Es posible, que este tipo de material se pueda utilizar en la industria del empaque, en la industria pesquera artesanal. 


\section{BIBLIOGRAFÍA}

1. Afirin, Y. H. y Yusuf, Y. (2013). Mycelium fibers as new resource for environmental sustainability. Procedia Engineering, 53(1), 504-508. https://doi: 10.1016/j.proeng.2013.02.065.

2. Amtislavski, P.; Yang, Z. (2017). White, MD Publicación de la solicitud de patente de los Estados Unidos; Patente y marca comercial de EE. UU. Oficina: Washington, DC, EE. UU. https://patents.google.com/patent/WO2017132523A1/en

3. Appels, F. V. W., Camere, S., Montalti, M., Karana, E., Jansen, K. M. B., Dijksterhuis, J., Krijgsheld, P y Wösten, H. (2019). Fabrication factors influencing mechanical, moisture-and waterrelated properties of mycelium-based composites. Materials \& Design, 161(6), 64-71. https://doi.org/10.1016/j.matdes.2018.11.027

4. Attias, N., Danai, O., Ezov, N., Tarazi, E. y Grobman, Y.J. (2017). Developing novel applications of micelyum based biocomposite materials for desing and architecture. Building with bio-based materials: Best practice and performance. https://tcode.net.technion.ac.il/files/2017/09/FP1303-paper-NoamAttias_31_7_17.pdf

5. Attias, N., Danai, O., Ezov, N., Tarazi, E., Y Grobman, Y. J. 2017. Developing novel applications of mycelium based bio- composite materials for design and architecture. En Attias, N., Danai, O., Ezov, N., Tarazi, E., Y Grobman, Y. J. (Eds). Pp 1-10. Building with bio-based materials: best practice and performance specification. Technion Israel Institute of Technology, Haifa, Israel.

6. Attias, N.; Danai, O.; Tarazi, E,; Pereman, I.; Grobman, Y.J. 2019. Implementing bio-design tools to develop mycelium-based products. The Design Journal. An International Journal for All Aspects of Design 22: (1): 1647-1657

7. Basu, N. y Nath, S. 2009 Fundamentals of biomaterials and biocompatibility. En Basu, B.; Dhirendra, K. y Kumar, A. (Comp.). Advanced Biomaterials. Fundamentals, processing and applications. Jonh Wiley and Sons. New Jersey. Pp. 3-16

8. Bidegain, M. A., Cubitto, M. A. y Curvetto, N. R. (2015). Optimization of the yield of Lingzhi or Reishi medicinal mushroom, Ganoderma lucidum (Higher basidiomycetes), Cultivated on a sunflower seed hull substrate produced in Argentina: effect of olive oil and copper. International Journal of Medicinal Mushrooms, 17(11), 1095-1105. DOI: 10.1615 / IntJMedMushrooms.v17.i11.100

9. CONAFOR, (2010). Consideraciones tecnológicas en la protección de la madera. SEMARNAT, Jalisco, México. https://www.conafor.gob.mx/biblioteca/Consideraciones-tecnologicas-de-la-madera.pdf

10. Danay, O., Ezov, N., Yosef, E. y Levanon, D. (2012). Recycling Lygnocelullosic Wastes for Mushroom Production and Cattle Feed. In: Proceedings of the 17th Congress of International Society for Mushroom Science. China: Agricultural Press, 18, 875-880

11. Do Prado, C. P., Manion, B. A., Seetharaman, K., Deschamps, F. C., Barrera Arellano, D., y Block, J. M. (2013). Relationship between antioxidant properties and chemical composition of the oil and the shell of pecan nuts [Carya illinoinensis (Wangenh) C. Koch]. Industrial Crops and Products, 45, 64-73. https://doi.org/10.1016/j.indcrop.2012.11.042 
12. Feijóo, K., Bermúdez, S., Rebolledo, H., Figueroa, J. M., Zamora, P y Naranjo, L. (2021). Fungal mycelium-bioproducts development: A new material culture and its impact on the transition to a sustainable economy. Bionatura Latin American Journal of Biotechnology and Life Sciences, 6(1). http://dx.doi.org/10.21931/RB/2021.06.01.29

13. Forero, C. A., Camargo, G y Sierra, F. E. (2014). Modelos de compresión aplicados al proceso de densificación de combustibles sólidos binarios carbón-madera. Iteckne, 11(2). DOI: https://doi.org/10.15332/iteckne.v11i2.73

14. Fricker, M., Boddy, L. y Bebber, D. (2007). Biology of the Fungal Cell. Springer. https://link.springer.com/book/10.1007/978-3-540-70618-2

15. García, D y Serrano, H. (2015). Los nogales de la barra del Meztitlán-Hidalgo. Tecno-agro. https://tecnoagro.com.mx/no.-103/los-nogales-de-la-barranca-de-meztitlan-hidalgo-la-barranca-demeztitlan

16. Girometta, C., Picco, A. M., Baiguera, R. M., Dondi, D., Babbini, S., Cartabia, M., Pellegrini, L. y Savino, E. (2019). Physico-Mechanical and termodynamic properties of micelium-based biocomposites: A review. Sustainability, 11(1), 281. https://doi.org/10.3390/su11010281

17. Glass, N. L., Rasmussen, C., Roca, M. G. y Read, N. D. (2004). Hyphal homing, fusion and mycelial interconnectedness. Trends in Microbiology. 12(3), 135-141. DOI: 10.1016 / j.tim.2004.01.007

18. González-Matute, R., Finglas, D., Delmastro, S. y Curvetto, N. (2002). Sunflower seed hulls as a main nutrient source for cultivating Ganoderma lucidum. Mycology Applicate International, 14(2), 1924. https://www.redalyc.org/pdf/685/68514202.pdf

19. Griffith, L.G. (2000). Polymeric biomaterials. Acta materialia, 48, 263-277. https://doi.org/10.1016/S1359-6454(99)00299-2

20. Haneef, M., Ceseracciu, L., Canale, C., Bayer, I. S., Heredia-Guerrero, J.A. y Athanassiou, A. (2017). Advanced materials from fungal mycelium: Fabrication and tuning of physical properties. Sci. Rep. 7, 4129. https://www.nature.com/articles/srep41292

21. Islam, M. R., Tudryn, G., Bucinell, R., Schadler, L. y Picu, R. C. (2017). Morphology and mechanics of fungal mycelium. Scientific Reports. 7. https://www.nature.com/articles/s41598-017$\underline{13295-2}$

22. Islam, M. R., Tudryn, G., Bucinell, R., Schadler, L. y Picu, R. C. (2018). Mechanical behavior of mycelium-based particulte composites. Journal of Materials Science, 53(24). 1671-1682. https://www.researchgate.net/publication/327065922_Mechanical_behavior_of_myceliumbased_particulate_composites

23. Jemison M., Olabisi, R. (2021). Biomaterials for human space exploration: A review of their untapped protential. Avta Biomaterialia, 128, 77-89.

24. Kuijk, S.J.V et al., (2017). Chemical changes and increased degradability of wheat straw and oak wood chips treated with rot fungi Ceriporiopsis subvermipora and Lenticulina edodes. Biomass and Bioenergy, 105(1), 381-391. https://doi.org/10.1016/j.biombioe.2017.07.003 
25. Lekounougou, S., Mounguengui, S., Dumarcay, S., Rose, C., Courty, P. E., Garbaye, J., Gérardin, P., Jacquot., J.P. y Gelhaye, E. (2008). Initial stages of Fagus sylvatica wood colonization by the whiterot basidiomycete Trametes versicolor. Enzymatic characterization. International Biodeterioration \& Biodegradation, 61(4), 287-293. https://doi.org/10.1016/j.ibiod.2007.06.013

26. Levelton B. (1975) "Combustion technology for the disposal and utilization of wood residue". Report EPS. 11 LEVELTON, BH \& ASSOCIATION Ltd. 1975. Tecnología de combustión para la eliminación y utilización de residuos de madera. (Elaborado para Environment Canada, Informe EPS 3AP-75-4.)

27. Matos, M. P., López, T. J., Lima, N. B., Griza, S., Rodríguez, S. F. y Marino, H. (2019). Production of biocomposites from the reuse of coconut powder colonized by Shiitake mushroom. Science and Agrotechnology. http://dx.doi.org/10.1590/1413-7054201943003819

28. Miles, G. P. y Chang, S. (1997). Mushroom biology, concise basic and current developments. Word scientific. http://files.shroomery.org/attachments/24154074Mushroom\%20Biology\%20Concise\%20Basics\%20And\%20Current\%20Developments\%20\%20Philip\%20G.\%20Miles,\%20Shu-Ting\%20Chang.pdf

29. Powrie, W. D., Wu, C.H., vicepresidente de Molund (1986). Sistemas de reacción de pardeamiento como fuentes de mutágenos y antimutagenos. Reinar. Perspectiva de salud, 67, 47-54. DOI.org/10.1016/j.Sc.2010.0037

30. Prodanović, S.; Milutinović, M. 2017. Some Applications of Biomaterials in the Automotive Industry. En Pellicer, E.; Nikolic, D.; Sort, J.; Baró, M;Zivic, F.; Grujovic, N.; Grujic,R.; Pelemis, S. (Comp.). Advances in Applications of Industrial Biomaterials. Springer. Suiza. Pp. 2-20

31. Rodríguez J., S. (2018). Material biobasado compuesto por el micelio de hongos descomponedores de madera y residuos agroindustriales. Revista Chilena de Diseño: creación y pensamiento, 3(5), 1-15. DOI: $10.5354 / 0719-837 x .2018 .50632$

32. Rodríguez, N. y Jaramillo, C. (2005). Cultivo de Hongos Medicinales en residuos agrícolas de la zona cafetera. Cenicafe, 28. https://www.cenicafe.org/es/publications/bot028.pdf

33. Sánchez, J. E. y Royse, D. J. (2001). La biología y cultivo de Pleurotus spp. Uteha-ECOSUR. https://www.researchgate.net/publication/256526787_Book_La_biologia_y_el_cultivo_de_Pleurotus_sp $\mathrm{p}$

34. Serret, N., Giralt, G. y Quintero M. (2016). Caracterización de aserrín de diferentes maderas. Tecnología química, 36(3). http://scielo.sld.cu/scielo.php?script=sci_arttext\&pid=S222461852016000300012

35. Sobal, M., Martínez-Carrera, D., Morales, P. y Roussos, S. (2007). Classical characterization of mushroom genetic resources from temperate and tropical regions of Mexico. Micología Aplicada Internacional, 19(1),15-23. https://www.redalyc.org/pdf/685/68519102.pdf

36. Sobal, M., Martínez-Carrera, D., Morales, P. y Roussos, S. (2007). Classical characterization of mushroom genetic resources from temperate and tropical regions of Mexico. Micología Aplicada Internacional, 19(1),15-23. https://www.redalyc.org/pdf/685/68519102.pdf 
37. Suárez, C. y Nieto, I. J. (2013). Cultivo Biotecnológico de macrohongos comestibles: una alternativa en la obtención de nutracéuticos. Revista Iberoamericana de Micología, 30(1), 1-8. http://www.reviberoammicol.com/2013-30/001008.pdf

38. Sun, W., Tajvidi, Mehdi., Hunt, G. C., Mc lntyre., G. y Gardner D. J. (2019). Fully Bio-Based Hybrid Composites Made of Wood Fungal Mycelium and Cellulose Nanofibrils. Scientific Reports, 9. 3766. DOI:10.1038/s41598-019-40442-8

39. Torres, A. M., Morales, J. C. y Atehortua, L. (2011). Efecto de nutrientes sobre la producción de biomasa del hongo medicinal Ganoderma lucidum. Revista Colombiana de Biotecnología, 13(1), 103 109. http://www.scielo.org.co/scielo.php?script=sci_arttext\&pid=S0123-34752011000100014

40. Yang, F., Ke, Y. y Kuo, S. (2000). Effect of fatty acids on the mycelial growth and polysaccharide formation by Ganoderma lucidum in shake flask cultures. Enzyme Microb Technol, 27, 295-301. DOI: 10.1016 / s0141-0229 (00) 00213-1

41. Zeller, P. y Zocher, D. (2012). Ecovative's breakthrougt biomaterials. Fungi, 5(1), 51-56. https://www.fungimag.com/spring-2012-articles/LR_Ecovative.pdf 